\title{
Research Article \\ Dynamic Change of Polymer in Rice Analogues and Its Effect on Texture Quality
}

\author{
Ke Zhang, Yang Tian, Chenglong Liu, and Wentong Xue \\ College of Food Science \& Nutritional Engineering, China Agricultural University, China \\ Correspondence should be addressed to Wentong Xue; wentongxueedu@163.com
}

Received 8 January 2020; Revised 12 March 2020; Accepted 20 March 2020; Published 8 April 2020

Academic Editor: Anjanapura V. Raghu

Copyright @ 2020 Ke Zhang et al. This is an open access article distributed under the Creative Commons Attribution License, which permits unrestricted use, distribution, and reproduction in any medium, provided the original work is properly cited.

\begin{abstract}
Macromolecules will leach from the inside of rice analogues (RA) to the external environment and form escaping substances (ES) when boiling in water for a long time. Some escaped substances adhere to the surface of cooked rice analogues (CRA) to form an adhesive layer (AL), which has an important impact on the cooking quality of RA. In this study, hydrocolloids and emulsifier were added and formed RA. Physicochemical, structural, and textural properties of ES, AL, and CRA samples were analyzed to study the effect of hydrocolloids on cooking quality of RA. The results showed that SA inhibited the leach of molecules, reduced MW of AL, decreased starch content of ES and AL, decreased shear viscosity of RA, and enhanced hydrogen bonding interactions. Ca ${ }^{2+}$ increased the dry matter content of CRA and AL, enhanced hydrogen bonding interactions of ES and CRA, and decreased MW of ES. Textural property results showed that the gelatinous properties of RA were enhanced after SA was added. The $\mathrm{Ca}^{2+}$ in the solution increased the adhesiveness of RA, while decreasing their elasticity. This study explained how hydrocolloids affect the texture properties of RA at a molecular level.
\end{abstract}

\section{Introduction}

Hydrocolloids are commonly used as thickeners, stabilizers, gelling agents, and emulsifiers in food products to improve the physicochemical and textural properties of products $[1,2]$. Hydrocolloids (such as sodium alginate and xanthan gum) can interact with starch, polysaccharides, and other substances in aqueous solution. A network structure is formed through covalent bonding of molecules and the intertwining of molecular chains, thereby increasing the viscosity of the solution or forming a gel [3]. In the presence of cations, xanthan gum (XG) forms intermolecular bonding thus diminishing interactions with other types of polysaccharides in the same solution [4]. SA reacts with $\mathrm{Ca}^{2+}$ to form intermolecular crosslinking, and gel strength increases with an increasing ionic strength. SA can form colloid after contacting with $\mathrm{Ca}^{2+}$ and is a widely used food additive. When the SA crosslinked with $\mathrm{Ca}^{2+}$, the flow of water molecules will be restricted, resulting in a gel with high water holding capacity. Different from other food gums, the colloid of SA and $\mathrm{Ca}^{2+}$ is not thermally reversible and usually exhibits good stability. Maitre et al. pointed out that the expansion ratio and water absorption index (WAI) increased with the incorporation of hydrocolloids whereas density, water absorption index (WSI), and hardness decreased upon extrusion $[5,6]$. Balakrishnan et al. found that nanocellulose could adhere to starch and form a stable network, and as it is capable of forming both intramolecular interaction with itself and strong intermolecular hydrogen bonding interaction with starch molecules, it can better interact with starch to elevate its properties [7-9].

Twin-screw extrusion technology is often used to enrich the diversity of rice products, improve nutritional value, and reduce production costs [10-12]. Extrusion of foods is an emerging technology for the food industries [13]. The dynamic propagation of cracks and fragmentation may occur in starch extrudates, which can be studied by the finite element method [14]. RA can be obtained according to many approaches by using brown rice as the main raw material, with the addition of various additives and other nutrients using twin-screw extruders [15]. Additionally, emulsifiers have been used to improve textural quality and taste of RA [16-18]. The emulsifier can combine with amylose to form a spiral complex, which will delay crystallization of the starch 
molecule during the retrogradation process [19]. The formation of such complex does not only reduce the viscosity and play a role of lubrication in the extrusion process but also is beneficial to the formation and dispersion of products because it can prevent the retrogradation and coagulation of starch. It has been documented that the addition of the emulsifier caused an increase in the degree of gelatinization and decrease of water-soluble carbohydrate, alpha-amylase sensitivity, WAI, and adhesive for extrudates [20-22].

The formula is generally based on the comprehensive consideration of rice composition, cooking characteristics, and sensory quality [23]. There are three main components presented upon extrusion, namely, escaping substances (ES), adhesive layer (AL), and cooked rice analogues (CRA), whose physical and chemical properties and structural characteristics have an important impact on cooking quality. Many have reported extrusion parameters and conditions of various grain-based products, yet less focuses on the effect of additives on the escape rule and structural change of molecules in RA.

In this study, hydrocolloids and emulsifier have been added to raw material and RA has been formed by thinscrew extrusion; RA was then subjected to the boiling test; subsequently, physicochemical and structural properties of ES, AL, and CRA have been analyzed to study the effects of molecular escape and structural changes on the properties of RA.

\section{Results and Discussion}

\subsection{Effects of Different Additives on the Textural Properties of} the Extrudates. The textural properties of the rice analogues with SA, XG, and SE after soaking are shown in Figure 1(a). SA reduced the viscosity of the sample, which was mainly reflected in the results of adhesion and maximum adhesion force. SA is a hydrophilic colloid that has been used as a thickening and gelling agent in the food industry. Previous studies have reported that SA reacts with starch to inhibit its expansion, protect the integrity of granules, and cause pea starch apparent pasting viscosity to decrease [24, 25]. Under the action of $\mathrm{Ca}^{2+}$, the adhesiveness and maximum adhesiveness of SARA increased, which indicated that cation-induced gelation could change the textural properties by altering the adhesion force within molecules.

Hecht and Srebnik mentioned that alginate tended to aggregate and form alohysical gel in the presence of cations. Specifically, $\mathrm{Ca}^{2+}$ facilitates chain aggregation and gelation [26]. XG reduced the sample height after hydrolysis, mainly due to the fact that XG was known to restore the mechanical properties of pregelatinized starch by forming a tighter network structure within CRA. SE can reduce the elasticity of the sample, which is related to the emulsification, ionic dispersion, and aging resistance properties [27]. The texture properties of the cooked samples (SARA, NARA) are shown in Figure $1(\mathrm{~b}) . \mathrm{CaCl}_{2}$ increased the adhesiveness and maximum adhesiveness of samples, while decreasing their elasticity. SA increased elasticity, chewiness, and extrusion work. Compared with soaked samples, the cohesion, elasticity, che- winess, height, and hardness of samples cooked with boiling water increased.

2.2. Analysis of Physical and Chemical Properties. The dry matter contents of ES, AL, and CRA are shown in Figure 2. SA increased the dry matter content of CRA and decreased that of ES and AL. SA could inhibit the dissociation of starch granules [28], resulting in retention of more ES in granules. $\mathrm{CaCl}_{2}$ increased the dry matter content of CRA and $\mathrm{AL}$ and decreased the dry matter content of ES. It showed that $\mathrm{CaCl}_{2}$ was helpful to the accumulation of the dry matter. In sum, the addition of $\mathrm{CaCl}_{2}$ in the presence of SA changed the aggregation behavior of the ES.

The results of starch content in Figure 2 showed that the starch content of ES and AL of NARA was higher than that of CRA after distilled water treatment. The addition of SA resulted in a decrease in starch content of ES and AL. $\mathrm{CaCl}_{2}$ treatment decreased the starch content of the AL of NARA, had no significant effect on the starch content of the ES, and reduced the starch content of CRA.

The results of amylose content are shown in Figure 2. The thickening effect of SA was beneficial to the aggregation of amylose, leading to the selective adsorption of amylose on CRA. $\mathrm{CaCl}_{2}$ treatment resulted in more amylose content of $\mathrm{AL}$ than that of ES. The addition of $\mathrm{CaCl}_{2}$ in the presence of SA made amylose content change more greatly.

The results of particle size distribution in Figure 2 showed that the particle size is in an ascending order: ES $>$ AL $>$ CRA. The ES contained narrower, more concentrated, and smaller particle size distribution than the AL. $\mathrm{CaCl}_{2}$ and SA had little effect on the size distribution. $\mathrm{CaCl}_{2}$ led to the increase of the particle size in the ES and CRA samples and the increase of the particle size of the AL. SA had led to an increased in particulate size of CRA. The presence of $\mathrm{SA}$ and $\mathrm{CaCl}_{2}$ facilitates the formation of $\mathrm{AL}$ on the surface of CRA.

The rheological properties are shown in Figure 3. According to the shear rate-viscosity curve, SA resulted in a decrease in the viscosity, and $\mathrm{CaCl}_{2}$ led to the increase in the viscosity of NARA. It has been pointed out that salt has a significant effect on starch gelatinization [29]. $\mathrm{CaCl}_{2}$ led to a decrease in the viscosity in the AL sample. These is a dramatic decrease in viscosity when the shear rate is at $50-150 \mathrm{~s}^{-1}$ for all samples. Although SA led to an increase in viscosity, when in the presence of $\mathrm{CaCl}_{2}$, the solution presents the highest viscosity.

SA reduced the storage modulus of the ES, while salt led to the maximum storage modulus of the ES of NARA. $\mathrm{CaCl}_{2}$ led to a decrease in the storage modulus of the AL. The storage modulus of CRA increased significantly due to the presence of SA and $\mathrm{CaCl}_{2}$. SA reduced the loss modulus of the ES, while $\mathrm{CaCl}_{2}$ led to the largest loss modulus of the ES of NARA. $\mathrm{CaCl}_{2}$ led to a decrease in the loss modulus of the AL. The combination of $\mathrm{CaCl}_{2}$ and SA resulted in a significant increase in the loss modulus of CRA.

2.3. Analysis of Structural Properties. FTIR spectra are shown in Figure 4. The peak at $3000-4000 \mathrm{~cm}^{-1}$ shows different hydrogen bonds. It was shown that the addition of SA 


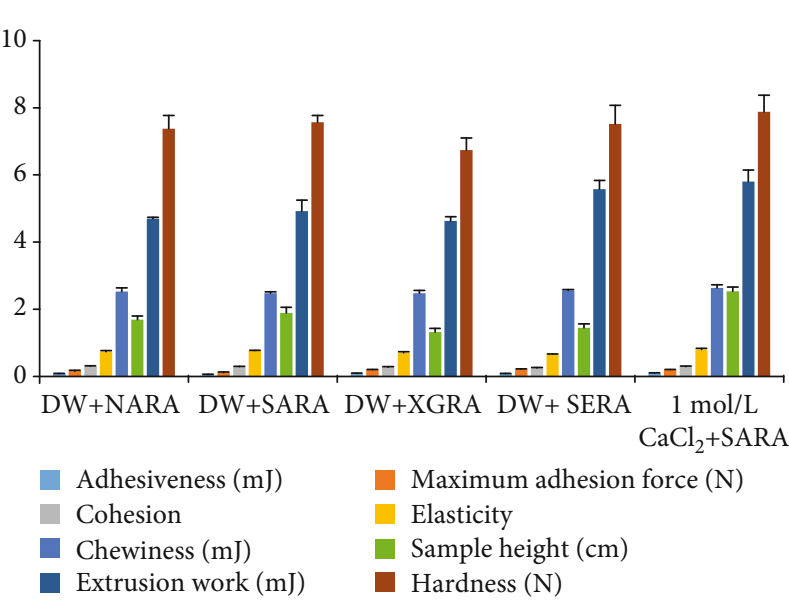

(a)

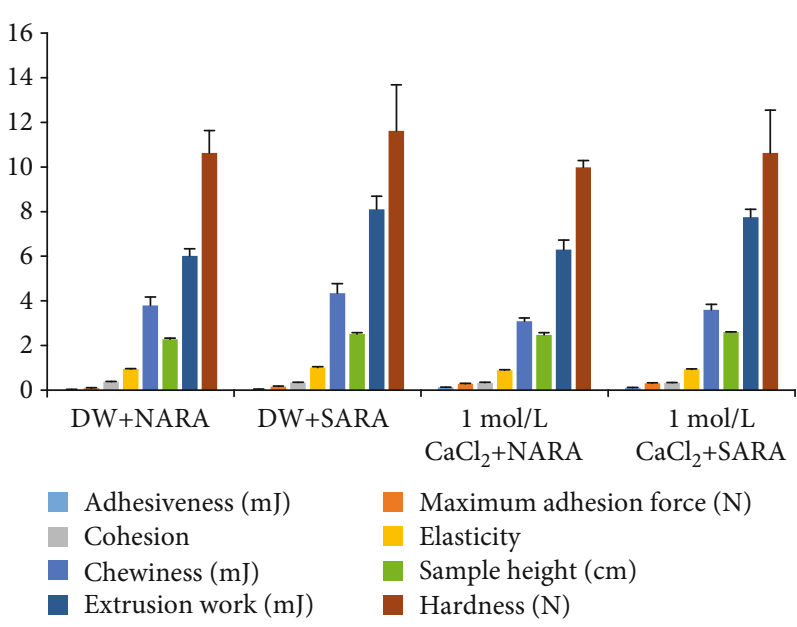

(b)

FIGURE 1: Textural properties of rice analogues: (a) soaking experiment; (b) boiling experiment. DW: distilled water; NARA: rice analogues with nonadditive; SARA: rice analogues containing sodium alginate; XGRA: rice analogues containing xanthan gum; SERA: rice analogues containing sucrose ester.

enhanced the hydrogen bonding interactions. Peaks near $2930 \mathrm{~cm}^{-1}$ characterize the stretching vibration of the C-H bond in the glucose unit $[30,31]$. SA weakened the stretching vibration of $\mathrm{C}-\mathrm{H}$ bond in the ES. The peak near $1625 \mathrm{~cm}^{-1}$, which characterizes the flexural vibration of $\mathrm{O}-\mathrm{H}$, is a typical absorption peak of starch and starch derivatives [30, 31]. SA increased the intensity of the peak at $1625 \mathrm{~cm}^{-1}$, indicating that the hydrogen bonding interactions in the molecule were enhanced. There are differences in FTIR spectra of the AL as compared with that of $\mathrm{ES}$. $\mathrm{CaCl}_{2}$ weakened the stretching vibration of the $\mathrm{C}-\mathrm{H}$ bond in the $\mathrm{AL}$. The complex action of $\mathrm{CaCl}_{2}$ and $\mathrm{SA}$ enhanced the bond of the hydrogen bonding interactions in the molecule. The FTIR spectra of CRA were different due to the treatment of $\mathrm{CaCl}_{2}$. $\mathrm{CaCl}_{2}$ increased the strength of the peak near $3000-4000 \mathrm{~cm}^{-1}$ and enhanced the hydrogen bonding interactions of CRA [32, 33].

2.4. Molecular Weight Distribution Analysis. The GPC spectrum of the AL sample is shown in Figure 5(a). The retention time and number of peaks decreased after SA was added. SA made MW of AL less than 3220. The result showed that the addition of SA has led to more small molecules adsorbed on the outer surface or AL of CRA, and the MW distribution was narrow. $\mathrm{CaCl}_{2}$ did not affect MW of the sample but has increased the number of peaks. The GPC spectra of the ES is shown in Figure 5(b). SA did not affect retention time and number of peaks. Under the action of $\mathrm{CaCl}_{2}$, the proportion of macromolecules (MW $=3220 \sim 1.65 \times 10^{8}$ ) decreased, while that of molecules of MW less than 3220 had increased. The GPC spectra of the CRA are shown in Figure 5(c). SA did not affect retention time of the peak but changed molecular weight distribution in CRA. The ratio of macromolecules in the MW range of $7.3 \times 10^{5} \sim 1.65 \times 10^{8}$ in DW+SARA samples is higher than that of molecules in similar MW range in the DW+NARA sample. The proportion of molecules increased in the MW range of $7.3 \times 10^{5} \sim 1.65 \times 10^{8}$ and decreased in the range of $3220 \sim 7.3 \times 10^{5}$ after adding SA.
$\mathrm{CaCl}_{2}$ in the presence of $\mathrm{SA}$ did not affect the molecular weight distribution of CRA.

2.5. Correlation Analysis. The results of correlation analysis between physicochemical properties and texture properties of products are shown in Table 1. ES content mainly affected the textural properties such as maximum adhesion force and extrusion work. The particle size distribution of ES mainly affected adhesion, maximum adhesion force, and sample height. The increase in dry matter content of ES led to the decrease of maximum adhesion force. The extrusion work of ES decreased with the increase of starch and amylose content. The increase in the average particle size of ES led to an increase of maximum adhesion force. Cohesion decreased with the increase in the mode of ES. The increase in particle size corresponds to accumulated particle size distribution of $10 \%$ (D10) of ES resulting in an increase in sample height. The increase in D25, D50, D75, and D90 of ES resulted in an increase of maximum adhesion force. The increase in D 90 of ES resulted in an increase in adhesiveness.

The components' content of AL mainly affected the maximum adhesion force. The particle size distribution of $\mathrm{AL}$ mainly affected adhesion, maximum adhesion force, cohesion, elasticity, chewiness, sample height, and hardness. The decrease in starch content and the increase in amylose content of the AL resulted in an increase in maximum adhesion force. The increase in the average particle size of AL led to an increase in elasticity and chewiness. The increase in D10 of $\mathrm{AL}$ resulted in an increase in cohesion and the decrease in sample height. The increase in D25 of AL resulted in a decrease in maximum adhesion force. The increase in D50 of $\mathrm{AL}$ resulted in a decrease in adhesiveness and maximum adhesion force. The increase in D75 of AL resulted in an increase in chewiness. The increase in D90 of AL resulted in an increase in elasticity, chewiness, and hardness.

The components' content of CRA mainly affected the maximum adhesion force, elasticity, and hardness. The 
ES
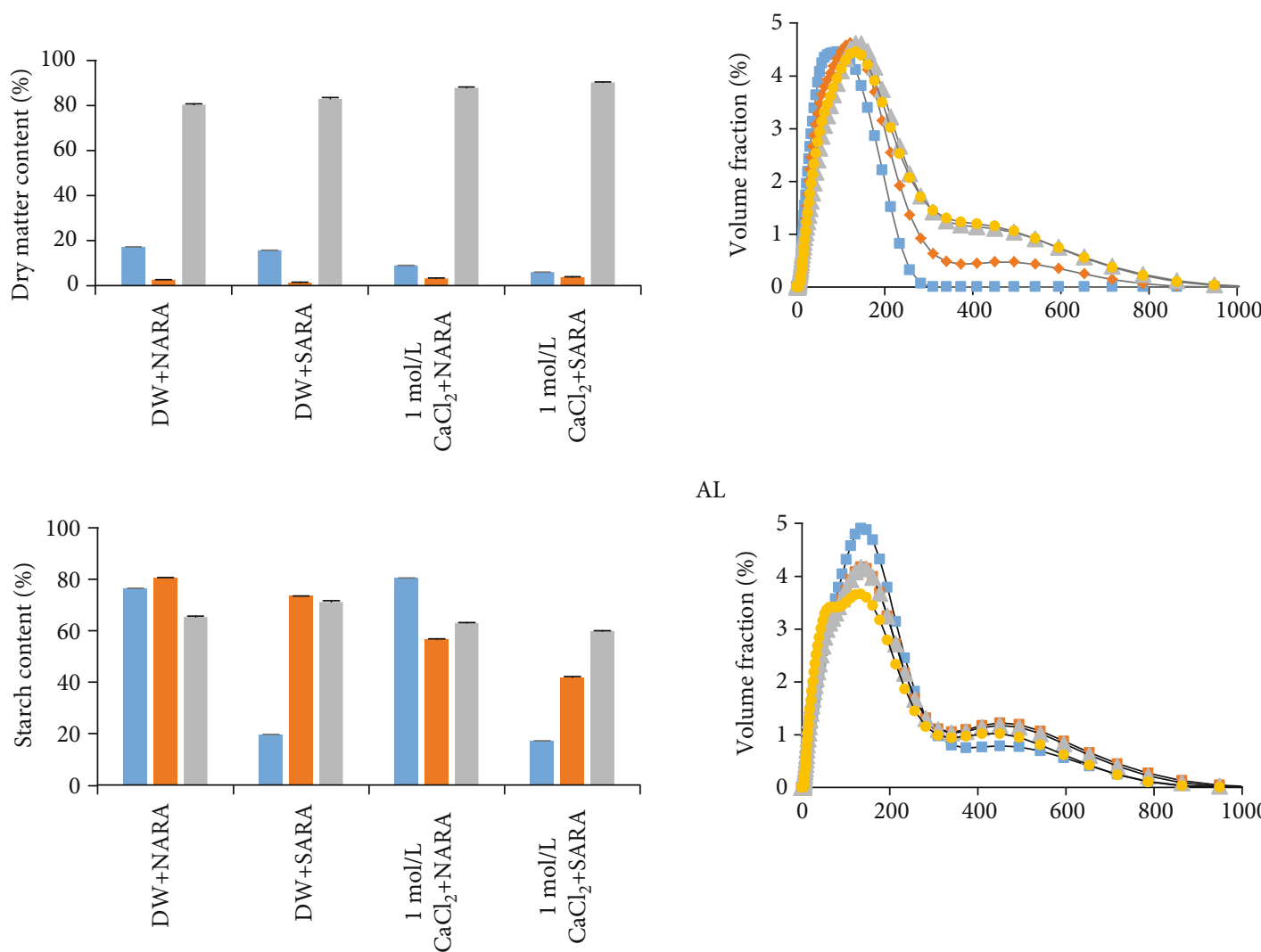

$\mathrm{AL}$
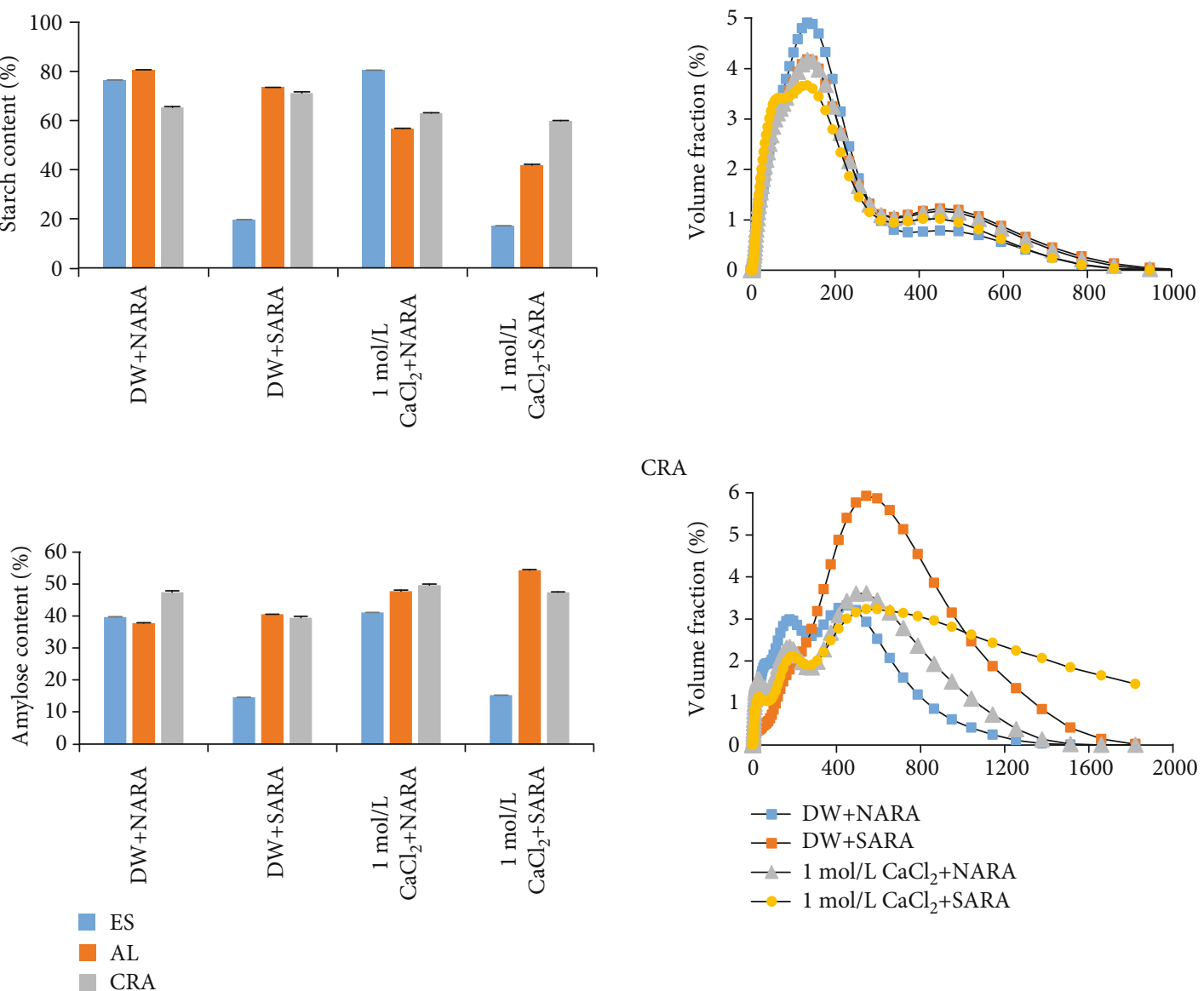

CRA

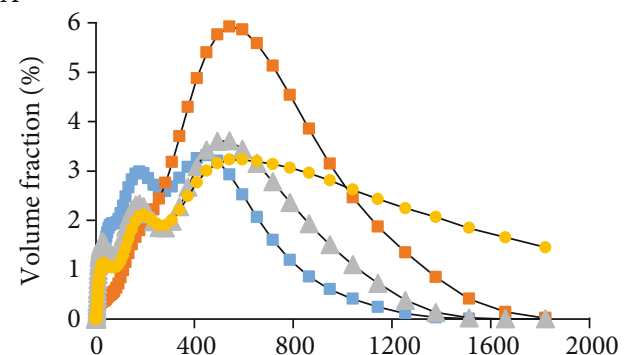

- DW+NARA

- - DW+SARA

$-1 \mathrm{~mol} / \mathrm{L} \mathrm{CaCl}_{2}+\mathrm{NARA}$

$-1 \mathrm{~mol} / \mathrm{L} \mathrm{CaCl}_{2}+\mathrm{SARA}$

FIGURE 2: Physicochemical properties of rice analogues. ES: substances escaping from rice analogues; AL: adhesive layer on the cooked rice analogues; CRA: cooked rice analogues; DW: distilled water; NARA: rice analogues with nonadditive; SARA: rice analogues containing sodium alginate.

particle size distribution of CRA mainly affected the height of the sample and the extrusion work. The increase in dry matter content of CRA led to an increase in maximum adhesion force. The increase in amylose content of CRA led to a decrease in elasticity and hardness. The extrusion work increased with the increase of the average particle size, D50, and D70 of CRA. The increase in mode led to the increase in sample height of CRA. The extrusion work increases with the increase in the average particle size of CRA.

\section{Materials and Methods}

3.1. Materials. Rice flour was provided by Henan Huangguo Grain Industry Co., Ltd. (stock code 831357). All the reagents used for the study were AR grade.

3.2. Extrusion. $600 \mathrm{~g}$ rice flour, $0.5 \%(w / w)$ additive, and $150 \mathrm{~g}$ distilled water were mixed thoroughly and processed by twin-screw extrusion equipment. RA for the experimental 

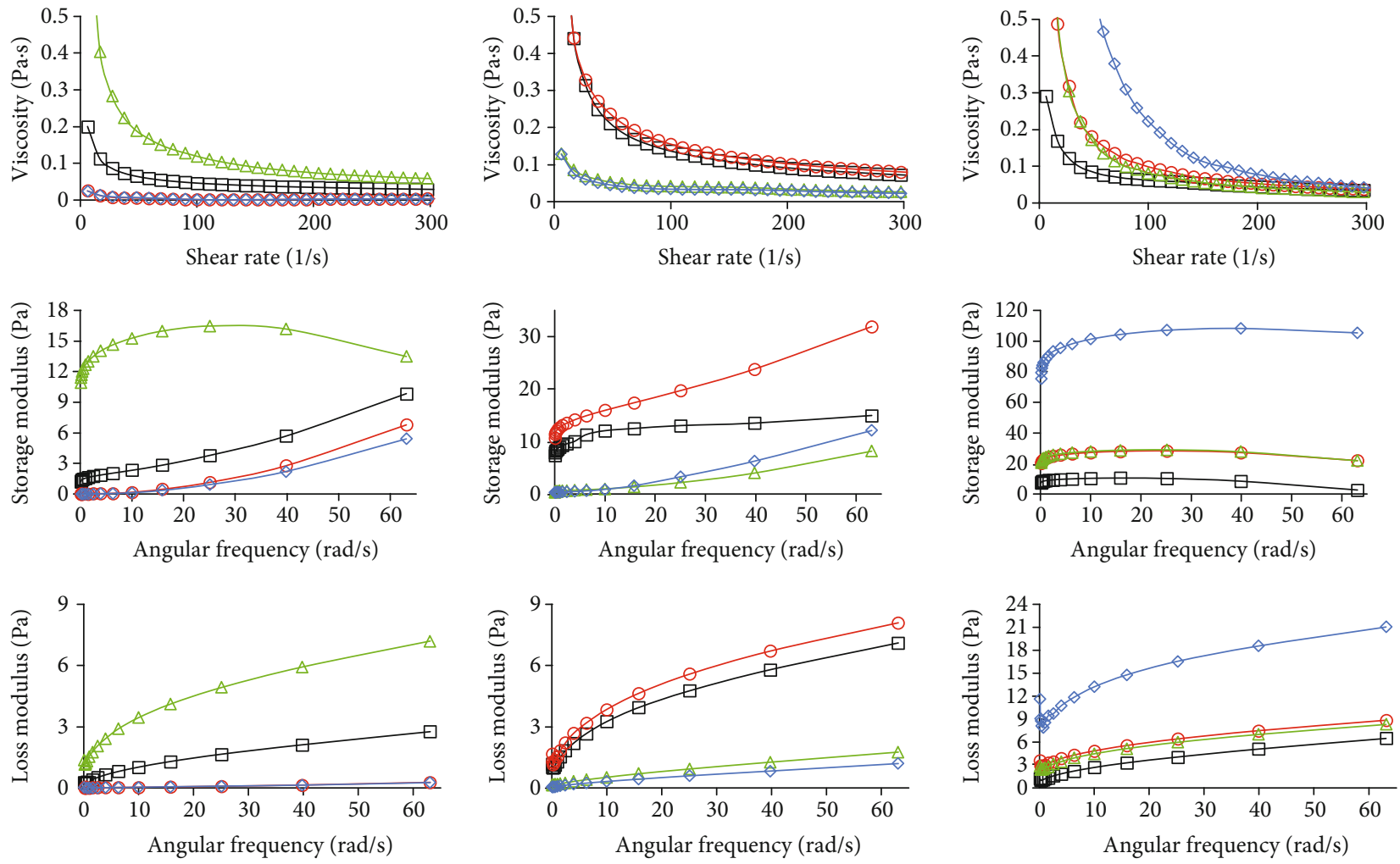

Distilled water +rice analogues with non-additive (NARA) $\triangle 1 \mathrm{~mol} / \mathrm{LCaCl}_{2}+\mathrm{NARA}$

Distilled water+rice analogues containing sodium alginate (SARA) $\diamond 1 \mathrm{~mol} / \mathrm{L} \mathrm{CaCl}_{2}+\mathrm{SARA}$

Figure 3: Rheological properties. (a) Escaping substances. (b)Adhesive layer. (c) Cooked rice analogues.
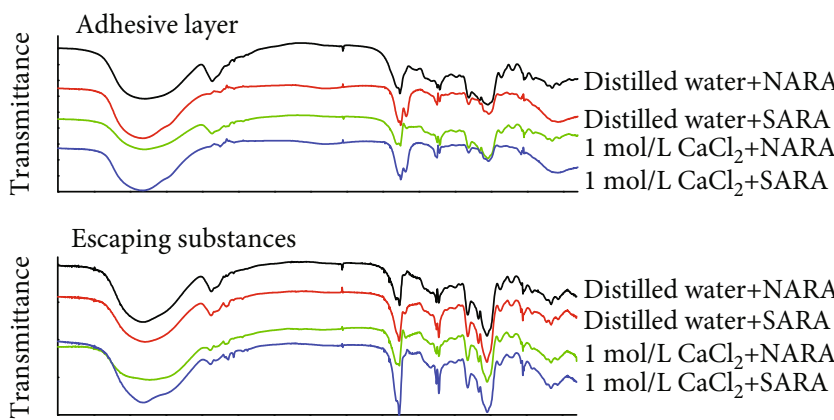

Cooked rice analogues

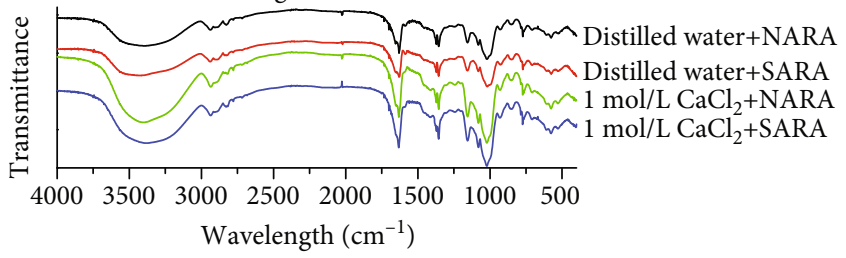

FIGURE 4: Fourier transform infrared spectroscopy of rice analogues. NARA: rice analogues with nonadditive; SARA: rice analogues containing sodium alginate.

study were obtained by dehydrating the extrudates at 25 for 48 hours and then drying them at 190 for 90 seconds. The twin-screw extruder used in the experiment consists of four parts: feed hopper, solid transport zone, melting, and melt pumping. The temperatures of the four regions during processing are $50,70,110$, and $85^{\circ} \mathrm{C}$, respectively. RA containing $\mathrm{SA}, \mathrm{XG}$, and sucrose ester (SE) are abbreviated as SARA, XGRA, and SERA. RA with nonadditive are abbreviated as NARA.

3.3. Dry Matter Content (DMC). The dry matter content was determined in A and B groups. The solution in group A was distilled water, and the solution in group B was $1 \mathrm{~mol} / \mathrm{L}$ $\mathrm{CaCl}_{2}$. Samples of about $10 \mathrm{~g}$ (SARA, NARA) were treated in $100 \mathrm{~mL}$ boiling distilled water for 4 minutes, and then, solid (S1) and liquid (L1) were separated by a $1 \mathrm{~mm}$ pore size filter. L1 was centrifuged $(6000 \mathrm{r} / \mathrm{min}, 15 \mathrm{~min})$ after cooling to $25^{\circ} \mathrm{C}$ and freeze-dried for $48 \mathrm{~h}$. After crushing, the ES of RA was obtained. S1 was immersed in distilled water at $25^{\circ} \mathrm{C}$ and stirred rapidly with a glass rod, and then, liquid (L2) and solid (S2) were separated with a $1 \mathrm{~mm}$ pore size filter. The separation operation was repeated three times. Precipitation of L2, gathered by centrifugation $(6000 \mathrm{r} / \mathrm{min}$, $15 \mathrm{~min}$ ), was freeze-dried for $48 \mathrm{~h}$ and pulverized for the study of the AL. CRA were prepared by freeze-drying S2 for $48 \mathrm{~h}$ and crushing. The ES, AL, and CRA in group B were prepared by replacing the solution in group A with calcium chloride and repeating the operation.

3.4. Starch and Amylose Content. Starch content of samples was determined by a starch content detection kit (Beijing Solarbio Science \& Technology Co., Ltd.). The soluble sugar 

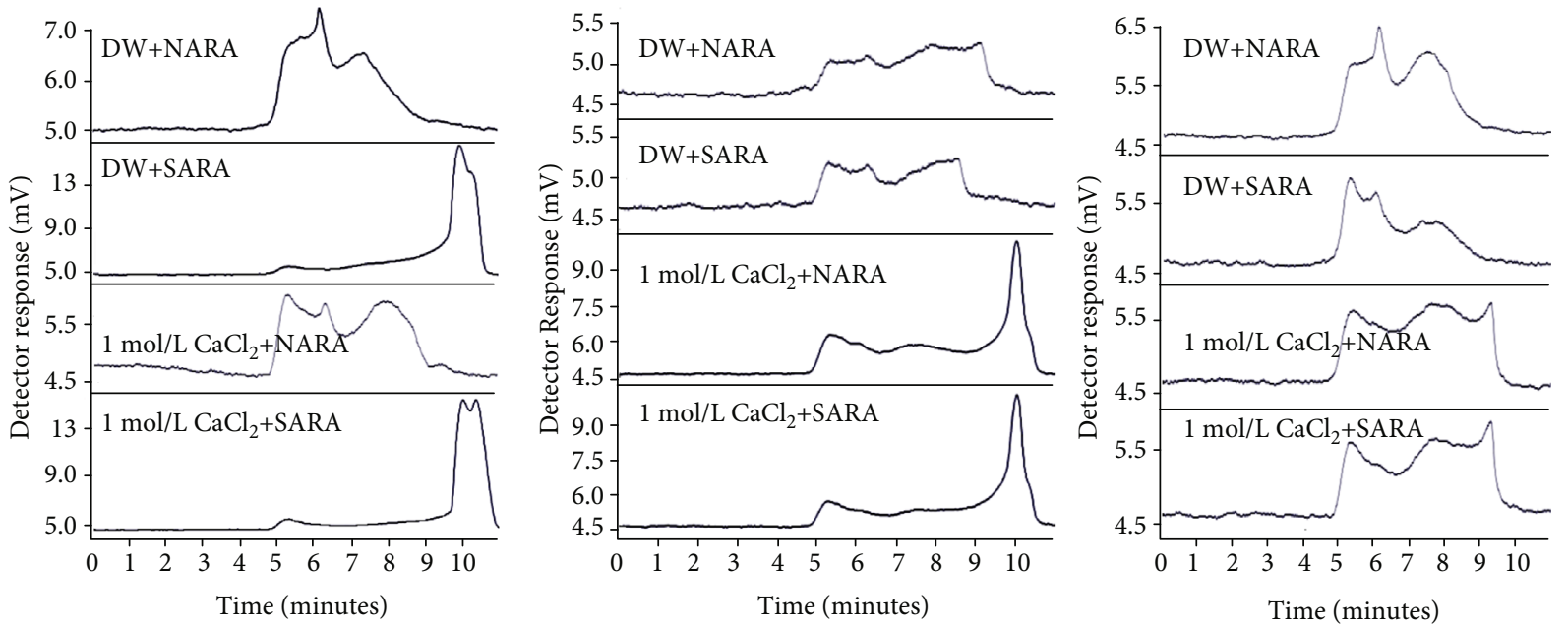

\begin{tabular}{cccccc}
\hline Time (minutes) & 5 & 6 & 7 & 8 & 9 \\
\hline Molecular weight & $1.65 \mathrm{E}+08$ & $1.10 \mathrm{E}+07$ & $7.30 \mathrm{E}+05$ & $4.85 \mathrm{E}+04$ & $3.22 \mathrm{E}+03$ \\
\hline
\end{tabular}

(a)

(b)

(c)

FIGURE 5: Molecular weight distribution. DW: distilled water; NARA: rice analogues with nonadditive; SARA: rice analogues containing sodium alginate. Adhesive layer. Escaping substances. Cooked rice analogues.

and starch in the sample were separated by $80 \%$ ethanol. Through the hydrolysis of acids, the starch was decomposed into glucose, whose content was determined by anthrone colorimetry. Finally, the content of starch was calculated. Each value was the mean of three replicates.

Amylose content of samples was determined by an amylose content kit (Nanjing Jiancheng Bioengineering Institute). The soluble sugar and starch in the sample were separated by $80 \%$ ethanol. According to the strong absorption peak of the complex formed by amylose and iodine under $620 \mathrm{~nm}$ ultraviolet light, the content of amylose was determined by an ultraviolet spectrophotometer. Each value was the mean of three replicates.

3.5. Particle Size and Distribution [2]. The particle size distribution of sample was analyzed using a laser particle size analyzer (LS230, Beckman, USA). Each value was the mean of three replicates.

3.6. Rheological Properties [34]. The dynamic and static rheological properties of $5 \%(w / w)$ sample suspension at $25^{\circ} \mathrm{C}$ were measured by a rheometer. $0 \sim 65 \mathrm{rad} / \mathrm{s}$ of the angular frequency omega and $2 \%$ of strain were carried out in the dynamic rheological test. The change of viscosity with the increasing shear rate $\left(1.0 \sim 300 \mathrm{~s}^{-1}\right)$ is recorded for static rheological test analysis.

\subsection{Structural Characterization (Fourier Transform Infrared} (FTIR)) [35]. FTIR spectra were collected for potato flour and their paste using an FTIR spectrometer (SPECTRUM 100, PerkinElmer, America). 1-2 mg samples were blended with $200 \mathrm{mg} \mathrm{KBr}$ and pressed into tablets before measure- ment. Spectra were collected at a resolution of $4 \mathrm{~cm}^{-1}$ and at an average of 4 scans per sample.

3.8. Gel Permeation Chromatography (GPC). The molecular weight (MW) distribution of samples was determined using GPC, following a previously published method [36]. The sample was prepared by mixing $25 \mathrm{mg}$ of ES, AL, and CRA with $5 \mathrm{~mL}$ ultrapure water, respectively. Ultrasound treatment for 5 minutes was performed to assist dissolution of analysts. The solution was treated with water bath at $99^{\circ} \mathrm{C}$ for $1 \mathrm{~h}$ (oscillating per 15 minutes) and then cooled to room temperature. After dissolution, the solution was centrifuged at $25^{\circ} \mathrm{C}(6000 \mathrm{r} / \mathrm{min}, 15 \mathrm{~min})$; the supernatant was filtrated by a nylon microporous membrane $(0.22 \mu \mathrm{m})$. GPC measurements were performed using an Agilent 1260 Infinity LC system (size exclusion chromatography/gel permeation chromatography) equipped with a refractive index detection. Analysts of interest were separated on Aqueous and Polar Organic GPC/SEC Columns (Agilent Technologies, Santa Clara, CA, U.S.A.) using DI water as the mobile phase at a flow rate of $1 \mathrm{~mL} / \mathrm{min}$ at $35^{\circ} \mathrm{C}$.

3.9. Textural Properties. For the soaking experiment, samples of about $5 \mathrm{~g}$ (SARA, XGRA, SERA, and NARA) were placed in a beaker. After adding $10 \mathrm{~mL}$ boiling DI water, the beaker mouth was sealed with fresh-keeping film immediately. After 10 minutes, the solid was filtered out by a sieve with a diameter of $1 \mathrm{~mm}$. The solid was cooled in distilled water at $25^{\circ} \mathrm{C}$ for 5 minutes and determined by a texture analyzer.

For the boiling experiment, samples of about $10 \mathrm{~g}$ (SARA, NARA) were placed in $300 \mathrm{~mL}$ boiling distilled water for 4 minutes; then, solid was determined and filtered out with a $1 \mathrm{~mm}$ pore sieve and cooled in distilled water at $25^{\circ} \mathrm{C}$ for 5 
TABLE 1: Relevance analysis results.

\begin{tabular}{|c|c|c|c|c|c|c|c|c|}
\hline & Adhesiveness & $\begin{array}{l}\text { Maximum } \\
\text { adhesion } \\
\text { force }\end{array}$ & Cohesion & Elasticity & Chewiness & $\begin{array}{l}\text { Sample } \\
\text { height }\end{array}$ & $\begin{array}{l}\text { Extrusion } \\
\text { work }\end{array}$ & Hardness \\
\hline \multicolumn{9}{|l|}{$\mathrm{AL}$} \\
\hline $\begin{array}{l}\text { Dry matter } \\
\text { content }\end{array}$ & NS & NS & NS & NS & NS & NS & NS & NS \\
\hline Starch content & NS & $-0.965^{*}$ & NS & NS & NS & NS & NS & NS \\
\hline Amylose content & NS & $0.960^{*}$ & NS & NS & NS & NS & NS & NS \\
\hline Mean & NS & NS & NS & $0.969^{*}$ & $0.983^{*}$ & NS & NS & NS \\
\hline Mode & NS & NS & NS & NS & NS & NS & NS & NS \\
\hline D10 & NS & NS & $0.975^{*}$ & NS & NS & $-0.977^{*}$ & NS & NS \\
\hline D25 & NS & $-0.984^{*}$ & NS & NS & NS & NS & NS & NS \\
\hline D50 & $-0.997^{* *}$ & $-0.967^{*}$ & NS & NS & NS & NS & NS & NS \\
\hline D75 & NS & NS & NS & NS & $0.972^{*}$ & NS & NS & NS \\
\hline D90 & NS & NS & NS & $0.979^{*}$ & $0.951^{*}$ & NS & NS & $0.995^{* *}$ \\
\hline \multicolumn{9}{|l|}{ CRA } \\
\hline $\begin{array}{l}\text { Dry matter } \\
\text { content }\end{array}$ & NS & $0.993^{* *}$ & NS & NS & NS & NS & NS & NS \\
\hline Starch content & NS & NS & NS & NS & NS & NS & NS & NS \\
\hline Amylose content & NS & NS & NS & $-0.959^{*}$ & NS & NS & NS & $-0.974^{*}$ \\
\hline Mean & NS & NS & NS & NS & NS & NS & $0.997^{* *}$ & NS \\
\hline Mode & NS & NS & NS & NS & NS & $0.988^{*}$ & NS & NS \\
\hline D10 & NS & NS & NS & NS & NS & NS & NS & NS \\
\hline D25 & NS & NS & NS & NS & NS & NS & NS & NS \\
\hline D50 & NS & NS & NS & NS & NS & NS & $0.962^{*}$ & NS \\
\hline D75 & NS & NS & NS & NS & NS & NS & $0.964^{*}$ & NS \\
\hline D90 & NS & NS & NS & NS & NS & NS & NS & NS \\
\hline
\end{tabular}

ES: substances escaping from rice analogues; AL: adhesive layer on the cooked rice analogues; CRA: cooked rice analogues; mean: average particle size; mode: particle size with the largest number of particles; $D(X)$ : particle size corresponding to accumulated particle size distribution of $X \%$. *Significant correlation; ${ }^{* *}$ extremely significant correlation. NS: no correlation; significant positive and negative correlations were in bold and italic, respectively.

minutes. Samples of about $10 \mathrm{~g}$ (SARA, NARA) were placed in $300 \mathrm{~mL}$ boiling $1 \mathrm{~mol} / \mathrm{L} \mathrm{CaCl}$ for 4 minutes; then, solid was determined and filtered out with a $1 \mathrm{~mm}$ pore sieve and cooled in distilled water at $25^{\circ} \mathrm{C}$ for 5 minutes.

The textural properties were measured following the procedure by $\mathrm{Li}$ et al. with some modification by a texture analyzer, and the analysis was repeated five times [37]. Three rice analogues were tested on the stage. The test speed was $1 \mathrm{~mm} / \mathrm{s}$. The test mode was compression, and the compression ratio was $70 \%$.

3.10. Statistics. Analysis of variance (ANOVA) was conducted by the SPSS 19.0, and the significant difference between group means was analyzed by Duncan multiple range tests $(p<0.05)$. Drawing and picture modification were accomplished by Origin 9.0.

\section{Conclusions}

Physicochemical and structural properties of ES, AL, and CRA were investigated on the RA sample with the addition of SA and $\mathrm{CaCl}_{2}$. SA decreased the viscosity of the sample, which was mainly reflected in the results of adhesion and maximum adhesion force, while the addition of $\mathrm{CaCl}_{2}$ had the opposite effects. Cooking RA in boiling water was beneficial to improve the gel quality of RA and increased the cohesion, elasticity, chewiness, height, and hardness. SA and $\mathrm{CaCl}_{2}$ increased the dry matter content of CRA and decreased that of $\mathrm{ES}$. $\mathrm{CaCl}_{2}$ reduced dry matter content in solution. The addition of $\mathrm{CaCl}_{2}$ in the presence of SA changed the aggregation behavior of the ES. SA was beneficial to the aggregation of amylose, leading to the selective adsorption of amylose on CRA. SA and $\mathrm{CaCl}_{2}$ had opposite effects on shear viscosity and loss modulus. FTIR results show that SA enhanced hydrogen bonding interactions of ES and CRA. SA did not affect the MW distribution in ES and CRA samples but had caused a significant reduction in the presence of macromolecules in the $\mathrm{AL}$ sample. $\mathrm{CaCl}_{2}$ decreased macromolecular proportion in ES and CRA.

The correlation analysis showed that physicochemical and structural properties are closely related to texture of RA. The components' content of ES mainly affected the 


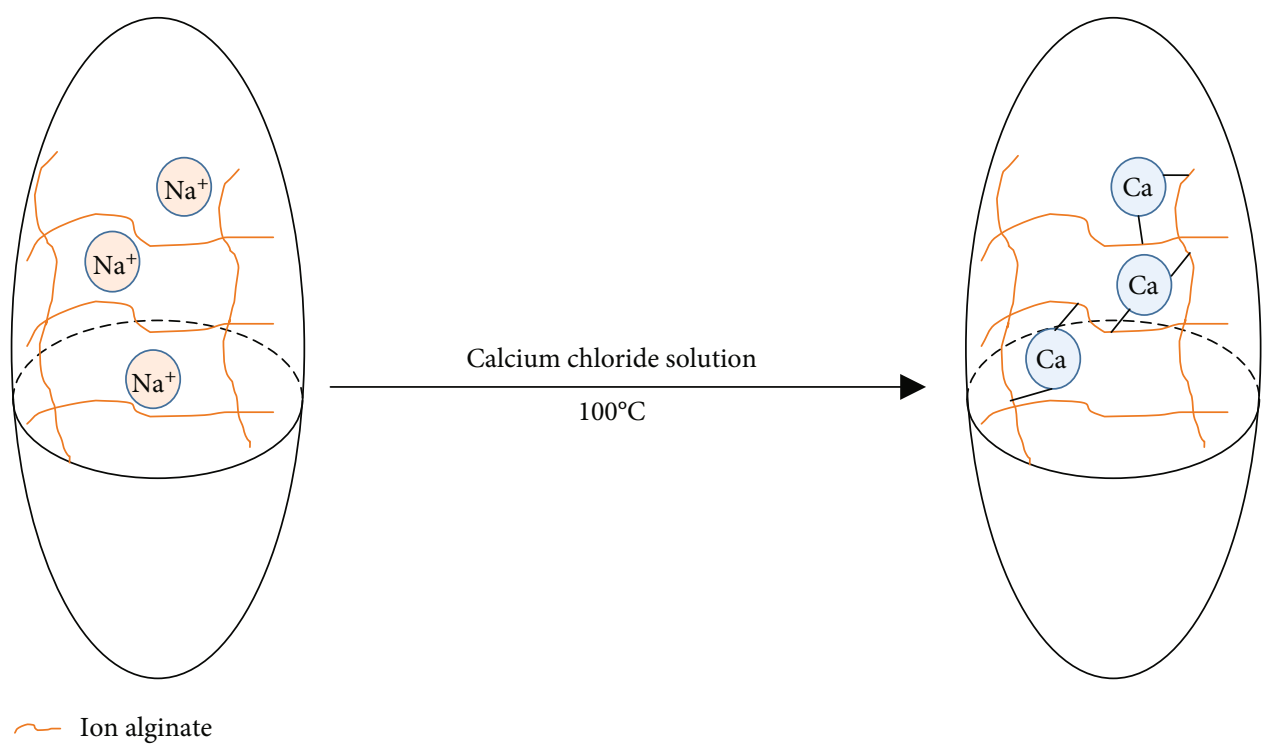

Figure 6: Complex effect of sodium alginate and calcium chloride on rice analogues.

maximum adhesion force and extrusion work. The components' content of AL mainly affected the maximum adhesion force. The particle size distribution of AL mainly affected adhesion and gel property. The components' content of CRA mainly affected the elasticity and hardness. The particle size distribution of CRA mainly affected the height of the sample and the extrusion work.

Based on the results stated above, a schematic diagram of how SA impacts textural properties of $\mathrm{xx}$ in the presence of $\mathrm{CaCl}_{2}$ is shown in Figure 6. Sodium alginate plays an active role in the network structure of rice analogues. When the samples were cooked with calcium chloride solution, ion exchange happened in the substance.

\section{Data Availability}

The research article data used to support the findings of this study are included within the article.

\section{Additional Points}

Highlights. (i) Hydrocolloid gel networks affect cooking properties of rice analogues. (ii) Molecules with high amylose content are more easily adsorbed on the surface of rice analogues. (iii) SA enhances hydrogen bonding interactions. (iv) Crosslinking of the SA allows an even distribution of molecular weight of the rice analogues.

\section{Conflicts of Interest}

The authors declare no conflict of interest.

\section{Authors' Contributions}

Ke Zhang and Wentong Xue conceived and planned experiments. Chenglong Liu and Yang Tian gave technical support and conceptual advice. Ke Zhang performed the experiments, analyzed data, and wrote the paper, and Wentong Xue proofed the paper. All authors discussed the results and implications and commented on the manuscript at all stages.

\section{Acknowledgments}

This research was funded by the National Key R\&D Program of China, grant number 2016YFD0401305.

\section{Supplementary Materials}

Sodium alginate (SA) inhibited the leach of molecules, reduced MW of the adhesive layer (AL), decreased starch content of escaping substances (ES) and AL, decreased shear viscosity of rice analogues (RA), and enhanced hydrogen bonding interactions. $\mathrm{Ca}^{2+}$ in the solution increased the dry matter content of cooked rice analogues (CRA) and AL, enhanced hydrogen bonding interactions of ES and CRA, and decreased MW of ES. The gelatinous properties of RA were enhanced after SA was added. $\mathrm{Ca}^{2+}$ in the solution increased the adhesiveness of RA and decreased their elasticity. (Supplementary Materials)

\section{References}

[1] N. Kaur, S. Sharma, D. N. Yadav, H. Bobade, and B. Singh, "Quality characterization of brown rice pasta supplemented with vital gluten and hydrocolloides," Agricultural Research, vol. 6, no. 2, pp. 185-194, 2017.

[2] Z. Lv, K. Yu, S. Jin et al., "Starch granules size distribution of sweet potato and their relationship with quality of dried and fried products," Starch - Stärke, vol. 71, no. 5-6, article 1800175, 2019.

[3] W. L. Peng, Y. You, P. Xie, M. Z. Rong, and M. Q. Zhang, "Adaptable interlocking macromolecular networks with homogeneous architecture made from immiscible single networks," Macromolecules, vol. 53, no. 2, pp. 584-593, 2020. 
[4] Q. Zhang, X. M. Hu, M. Y. Wu, M. M. Wang, Y. Y. Zhao, and T. T. Li, "Synthesis and performance characterization of poly(vinyl alcohol)-xanthan gum composite hydrogel," Reactive and Functional Polymers, vol. 136, pp. 34-43, 2019.

[5] A. Maitre, R. P. Rathod, and U. S. Annapure, "Effect of hydrocolloids and process parameters on the extrusion behavior of pearl millet grits," International Food Research Journal, vol. 24, no. 4, pp. 1538-1544, 2017.

[6] S. R. Bajaj and R. S. Singhal, "Effect of extrusion processing and hydrocolloids on the stability of added vitamin B12 and physico-functional properties of the fortified puffed extrudates," $L W T$, vol. 101, pp. 32-39, 2019.

[7] P. Balakrishnan, M. S. Sreekala, V. G. Geethamma et al., "Physicochemical, mechanical, barrier and antibacterial properties of starch nanocomposites crosslinked with pre-oxidised sucrose," Industrial Crops and Products, vol. 130, pp. 398-408, 2019.

[8] P. Balakrishnan, S. Gopi, S. M S, and S. Thomas, "UV resistant transparent bionanocomposite films based on potato starch/cellulose for sustainable packaging," Starch-Starke, vol. 70, no. 1-2, article 1700139, 2018.

[9] F. Mohanty and S. K. Swain, "Nano silver embedded starch hybrid graphene oxide sandwiched poly(ethylmethacrylate) for packaging application," Nano-Structures \& Nano-Objects, vol. 18, article 100300, 2019.

[10] J. M. Harper, "Food extrusion," Critical Reviews in Food Science and Nutrition, vol. 11, no. 2, pp. 155-215, 1978.

[11] H. Patil, R. V. Tiwari, and M. A. Repka, "Hot-melt extrusion: from theory to application in pharmaceutical formulation," AAPS PharmSciTech, vol. 17, no. 1, pp. 20-42, 2016.

[12] I. T. Ozbolat and M. Hospodiuk, "Current advances and future perspectives in extrusion-based bioprinting," Biomaterials, vol. 76, no. 37, pp. 321-343, 2016.

[13] M. S. Alam, J. Kaur, H. Khaira, and K. Gupta, "Extrusion and extruded products: changes in quality attributes as affected by extrusion process parameters: a review," Critical Reviews in Food Science and Nutrition, vol. 56, no. 3, pp. 445-475, 2016.

[14] J. Gao, Y. Wang, Z. Dong, and W. Zhou, "Structural and mechanical characteristics of bread and their impact on oral processing: a review," International Journal of Food Science and Technology, vol. 53, no. 4, pp. 858-872, 2018.

[15] A. Mishra, H. N. Mishra, and P. Srinivasa Rao, "Preparation of rice analogues using extrusion technology," International Journal of Food Science and Technology, vol. 47, no. 9, pp. 1789-1797, 2012.

[16] C. G. Dalbhagat, D. K. Mahato, and H. N. Mishra, "Effect of extrusion processing on physicochemical, functional and nutritional characteristics of rice and rice-based products: a review," Trends in Food Science \& Technology, vol. 85, pp. 226-240, 2019.

[17] S. Saadat, S. Movahhed, and H. Ahmadi Chenarbon, "Effect of guar and arabic gums on qualitative properties of extruded rice," Journal of Food Process Engineering, vol. 42, no. 2, article e12959, 2019.

[18] L. Agustina and C. Wibowo, "A preliminary study on the rehydration characteristics and cooking time of analog rice from the formulation of modified nagara bean flour through L. plantarum fermentation and sago starch," in IOP Conference Series: Earth and Environmental Science, Volume 255, 1st International Conference on Life and Applied Sciences for Sus- tainable Rural Development, Purwokerto, Central Java, Indonesia, November 2019.

[19] L. B. Deffenbaugh, "Emulsifier-carbohydrate interactions," in Food Emulsifiers and Their Applications, pp. 65-100, Springer, 2019.

[20] J. P. Wang, H. Z. An, Z. Y. Jin, Z. J. Xie, H. N. Zhuang, and J. M. Kim, "Emulsifiers and thickeners on extrusion-cooked instant rice product," Journal of Food Science and Technology, vol. 50, no. 4, pp. 655-666, 2013.

[21] S. Ranjbar, A. Basiri, A. H. Elhamirad, A. Sharifi, and H. A. Chenarbon, "Effect of hydrocolloids on physicochemical, sensory and textural properties of reconstructed rice grain by extrusion cooking technology," Journal of Food Measurement and Characterization, vol. 12, no. 3, pp. 1622-1632, 2018.

[22] Q. Yang, Z. Xiao, Y. Zhao, C. Liu, Y. Xu, and J. Bai, “Effect of extrusion treatment with different emulsifiers on the thermal stability and structure of corn starch," Czech Journal of Food Sciences, vol. 33, no. 4, pp. 464-473, 2015.

[23] S. B. Wahjuningsih and S. Susanti, "Chemical, physical, and sensory characteristics of analog rice developed from the mocaf, arrowroof, and red bean flour," IOP Conference Series: Earth and Environmental Science, vol. 102, article 012015, 2018.

[24] Y. Zhao, H. Chen, Y. Wang, and Q. Li, "Effect of sodium alginate and its guluronic acid/mannuronic acid ratio on the physicochemical properties of high-amylose cornstarch," Starch-Starke, vol. 68, no. 11-12, pp. 1215-1223, 2016.

[25] H.-S. Kim and J. N. BeMiller, "Effects of hydrocolloids on the pasting and paste properties of commercial pea starch," Carbohydrate Polymers, vol. 88, no. 4, pp. 1164-1171, 2012.

[26] H. Hecht and S. Srebnik, "Structural characterization of sodium alginate and calcium alginate," Biomacromolecules, vol. 17, no. 6, pp. 2160-2167, 2016.

[27] Z. u. Din, H. Xiong, Z. Wang, L. Chen, and I. Ullah, "Effects of sucrose fatty acid ester addition on the structural, rheological and retrogradation behavior of high amylose starch-based wood adhesive," International Journal of Adhesion and Adhesives, vol. 89, pp. 51-58, 2019.

[28] H. Zhuang, T. Feng, and J. N. BeMiller, "Effects of hydrocolloids on normal maize starch and its distarch phosphates," Food Hydrocolloids, vol. 96, pp. 231-245, 2019.

[29] W. Wang, H. Zhou, H. Yang, S. Zhao, Y. Liu, and R. Liu, "Effects of salts on the gelatinization and retrogradation properties of maize starch and waxy maize starch," Food Chemistry, vol. 214, pp. 319-327, 2017.

[30] K. Kaczmarska, B. Grabowska, T. Spychaj et al., "Effect of microwave treatment on structure of binders based on sodium carboxymethyl starch: FT-IR, FT-Raman and XRD investigations," Spectrochimica Acta Part A: Molecular and Biomolecular Spectroscopy, vol. 199, pp. 387-393, 2018.

[31] S. I. Rafiq, S. Singh, and D. C. Saxena, "Effect of heat-moisture and acid treatment on physicochemical, pasting, thermal and morphological properties of Horse Chestnut (Aesculus indica) starch," Food Hydrocolloids, vol. 57, pp. 103-113, 2016.

[32] T. Spychaj, M. Zdanowicz, J. Kujawa, and B. Schmidt, "Carboxymethyl starch with high degree of substitution: synthesis, properties and application," Polimery, vol. 58, no. 7/8, pp. 503511, 2013.

[33] Q. Jiang, W. Gao, X. Li, Z. Liu, L. Huang, and P. Xiao, “Synthesis and properties of carboxymethyl Pueraria thomsonii Benth. starch," Starch-Starke, vol. 63, no. 11, pp. 692-699, 2011. 
[34] A. K. Siroha, K. S. Sandhu, M. Kaur, and V. Kaur, "Physicochemical, rheological, morphological and in vitro digestibility properties of pearl millet starch modified at varying levels of acetylation," International Journal of Biological Macromolecules, vol. 131, pp. 1077-1083, 2019.

[35] S. V. Kumar, V. A. S. Kumar, and S. Kumar, "The influence of bound water on the FTIR characteristics of starch and starch nanocrystals obtained from selected natural sources.," Starch - Stärke, vol. 71, no. 5-6, article 1700026, 2018.

[36] K. Tao, C. Li, W. Yu, R. G. Gilbert, and E. Li, "How amylose molecular fine structure of rice starch affects functional properties," Carbohydrate Polymers, vol. 204, pp. 24-31, 2019.

[37] H. Li, S. Prakash, T. M. Nicholson, M. A. Fitzgerald, and R. G. Gilbert, "The importance of amylose and amylopectin fine structure for textural properties of cooked rice grains," Food Chemistry, vol. 196, pp. 702-711, 2016. 